\title{
Some New Exact Traveling Wave Solutions for the Generalized Benney-Luke (GBL) Equation with Any Order
}

\author{
Jun-Jie Wang ${ }^{1,2}$, Lian-Tang Wang ${ }^{2}$, Kuan-De Yang ${ }^{1}$ \\ ${ }^{1}$ Mathematics Department, Simao Teachers' College, Pu'er, China \\ ${ }^{2}$ Mathematics Department, Northwest University, Xi'an, China \\ Email: wangjunjie6688@sina.com
}

Received December 5, 2011; revised February 22, 2012; accepted February 29, 2012

\begin{abstract}
In the paper, a auxiliary equation expansion method and its algorithm is proposed by studying a second order nonlinear ordinary differential equation with a four-degree term. The method is applied to the generalized Benney-Luke (GBL) equation with any order. As a result, some new exact traveling wave solutions are obtained which singular solutions, triangular periodic wave solutions and jacobian elliptic function solutions. This algorithm can also be applied to other nonlinear wave equations in mathematical physics.
\end{abstract}

Keywords: Generalized Benney-Luke (GBL) Equation; Jacobian Elliptic Function; Singular Solution

\section{Introduction}

Nonlinear phenomena that appear in many areas of scientific fields such as solid state physics, plasma physics, fluid dynamics, mathematical biology and chemical kinetics can be modeled by partial differential equation. A broad class of analytical solutions methods and numerical solutions methods were used in handle these problems. The investigation of exact traveling wave solution to nonlinear equations plays an important role in the study of nonlinear physical phenomena. Various methods for seeking traveling wave solutions to nonlinear partial differential equations are proposed such as inverse scattering transform method [1], BÄacklund and Darboux transform [2-6], Hirota method [7], Lie group method $[8,9]$ and so on.

In the paper, we shall consider the following generalized Benney-Luke (GBL) equation [10]:

$$
\begin{aligned}
& \Phi_{t t}-\Delta \Phi+a \Delta^{2} \Phi-b \Delta \Phi_{t t}+p \Phi_{t}\left(\Phi_{x}\right)^{p-1} \\
& \left(\Phi_{x x}+\Phi_{y y}\right)+2\left[\left(\Phi_{x}\right)^{p} \Phi_{x t}+\left(\Phi_{y} \Phi_{y t}\right)\right]=0
\end{aligned}
$$

The paper is organized as follows. In Section 2, we present the auxiliary equation method and its algorithm. In Section 3, we present some exact traveling wave solutions of system (1.1). Finally some conclusion are given.

\section{The Auxiliary Equation Algorithm}

We outline our auxiliary equation algorithm:
Step I. For a given nonlinear wave equation with one physical field $u(x, y, t)$; in three variables $x, y, t$,

$$
F\left(u, u_{t}, u_{x}, u_{y}, u_{t x}, u_{t y}, u_{x y}, u_{x x x}, \cdots\right)=0
$$

We seek its special solution, traveling wave solution, in the form of

$$
u(x, y, t)=u(\xi), \xi=x+y-c t
$$

where $c$ is constant. Substitution (2.2) into (2.1) gives rise to a nonlinear ordinary differential equation

$$
G\left(u, u^{\prime}, u^{\prime \prime}, u^{\prime \prime \prime}, \cdots\right)=0
$$

Step II. To seek the traveling wave solution of (2.3), we assume that (2.3) has the solution in the form of

$$
u(\xi)=\sum_{i=0}^{n} a_{i} \phi^{i}(\xi)
$$

with the new variable $\phi(\xi)$ satisfying:

$$
\phi^{\prime}(\xi)^{2}=h_{0}+h_{2} \phi(\xi)^{2}+h_{4} \phi(\xi)^{4}
$$

$h_{j}$ are constants and $n$ is the integer to be determined later.

Step III. Determined the parameter $n$. Substituing Equa- tion (2.4) along with Equation (2.5) into Equation (2.3) and balancing the highest derivative term with the non- linear terms in the Equation (2.3), we then obtain the va- lue of $n$.

Step IV. Determine the parameters

$$
c, a_{i} ; h_{j}(i=0,1,2, \cdots, n, j=0,2,4) \text {, }
$$


Substitution Equation (2.4) along with Equation (2.5) into Equation (2.3) and setting the coefficients of all powers $\phi^{i} \phi^{j}(i=0,1,2, \cdots n, j=0,1)$ to zero, we will obtain a system of nonlinear algebraic equations (NAEs) with respect to the parameters

$$
c, a_{i} ; h_{j}(i=0,1,2, \cdots, n, j=0,2,4),
$$

By solving the NAEs if available, we can determine those parameters explicitly.

\section{Exact Traveling Wave Solutions for the Generalized Benney-Luke (GBL) Equation with Any Order}

By considering that the traveling wave solutions of (1.1) propagate in the direction of the positive $x$-axis, i.e. $\Phi(x, y, t)=u(x-c t, y)$ Ref. [10] gave the following traveling equation:

$$
\begin{aligned}
& \left(c^{2}-1\right) u_{x x}+\left(a-b c^{2}\right) u_{x x x x}-u_{y y}+\left(2 a-b c^{2}\right) u_{x x y y} \\
& -c\left[(p+2) u_{x}^{p} u_{x x}+p u_{x} u_{y}^{p-1} u_{y y}+2 u_{y}^{p} u_{x y}\right]=0
\end{aligned}
$$

In this paper, we will study the traveling wave solutions of (1.1) with the form:

$$
\begin{aligned}
& u(x, y, t)=\varphi(x+\beta y-c t)=\varphi(\xi), \\
& \xi=x+\beta y-c t
\end{aligned}
$$

where $c$ is the wave speed. Substituting it into (1.1) and noting (3.1), we have

$$
\begin{aligned}
& \left(c^{2}-1-\beta^{2}\right) \varphi_{\xi \xi} \\
& +\left[a-b c^{2}+\left(2 a-b c^{2}\right) \beta^{2}+a \beta^{4}\right] \varphi_{\xi \xi \xi \xi} \\
& -\frac{c(p+2)\left(1+\beta^{p+1}\right)}{p+1}\left[\varphi_{\xi}^{p+1}\right]_{\xi}=0
\end{aligned}
$$

Integrating (3.2) once with respect to $\xi$ and taking the integral constant as zero, we obtain

$$
\begin{aligned}
& \left(c^{2}-1-\beta^{2}\right) \varphi_{\xi}+\left[a-b c^{2}+\left(2 a-b c^{2}\right) \beta^{2}+a \beta^{4}\right] \varphi_{\xi \xi \xi} \\
& -\frac{c(p+2)\left(1+\beta^{p+1}\right)}{p+1}\left[\varphi_{\xi}^{p+1}\right]=0
\end{aligned}
$$

Letting

$$
\psi(\xi)=\frac{\mathrm{d} \varphi}{\mathrm{d} \xi}, \varphi(\xi)=\int \psi(\xi) \mathrm{d} \xi
$$

and writing

$$
\begin{aligned}
& A=\frac{1+\beta^{2}-c^{2}}{a-b c^{2}+\left(2 a-b c^{2}\right) \beta^{2}+a \beta^{4}}, \\
& B=\frac{c\left(1+\beta^{p+1}\right)}{(p+1)\left[a-b c^{2}+\left(2 a-b c^{2}\right) \beta^{2}+a \beta^{4}\right]}
\end{aligned}
$$

then from (3.4), we have that

$$
\frac{\mathrm{d}^{2} \psi}{\mathrm{d} \xi^{2}}=A \psi+(p+2) B \psi^{p+1}
$$

Let

$$
\psi(\xi)=\sqrt[p]{\Psi}
$$

We have

$$
\frac{1-p}{p} \Psi^{\prime 2}+\frac{1}{p} \Psi \Psi^{\prime \prime}-A \Psi^{2}+(p-2) B \Psi^{3}=0
$$

We expand the solution of Equation (3.6) in form of Equation (2.4). Substitution Equation (2.4) along with Equation (2.5) and balancing the highest order derivative term.

$\Psi \Psi^{\prime \prime}$ with nonlinear term $\Psi^{3}$ in Equation (3.6) gives $n=2$. Hence, we have

$$
\Psi(\xi)=a_{0}+a_{1} \phi(\xi)+a_{2} \phi^{2}(\xi)
$$

where $a_{0}, a_{1}, a_{2}$ are constant to be determined later; $\phi(\xi)$ satifiyed Equation (2.5).

We can get the following equation by Step IV

$$
f_{0}+f_{1} \phi+f_{2} \phi^{2}+f_{3} \phi^{3}+f_{4} \phi^{4}+f_{5} \phi^{5}+f_{6} \phi^{6}=0
$$

If $n=\frac{p m^{2}}{(p+1)^{2} l}$, we can get the following solution:

$$
\begin{aligned}
a_{2}= & -\frac{2 h_{4}\left(3 p^{3}+2-2 p\right)}{p^{3} B(p+2)} \\
a_{1}= & 0 \\
a_{0}= & \frac{A\left(3 p^{3}+2-2 p\right)}{2(p+2) B\left(p^{3}+1-p\right)} \\
h_{2}= & -\frac{A p^{3}}{2\left(p^{3}+1-p\right)} \\
h_{0}= & \frac{p^{6} A^{2}}{16 h_{4}\left(p^{3}+1-p\right)^{2}} \\
H(\xi)= & {\left[\frac{A\left(3 p^{3}+2-2 p\right)}{2(p+2) B\left(p^{3}+1-p\right)}\right.} \\
& \left.-\frac{2 h_{4}\left(3 p^{3}+2-2 p\right)}{p^{3} B(p+2)} \phi^{2}(\xi)\right]^{\frac{1}{p}}
\end{aligned}
$$

where

$$
\begin{aligned}
\phi^{\prime}(\xi)^{2}= & \frac{p^{6} A^{2}}{16 h_{4}\left(p^{3}+1-p\right)^{2}}-\frac{A p^{3}}{2\left(p^{3}+1-p\right)} \varphi^{2}(\xi) \\
& +h_{4} \varphi^{4}(\xi)
\end{aligned}
$$


Th 3.1. Suppose that

$$
\frac{p^{6} A^{2}}{16 h_{4}\left(p^{3}+1-p\right)^{2}}=h_{4},-\frac{A p^{3}}{2\left(p^{3}+1-p\right)}=-h_{4}
$$

If $h_{4}>0$, that Equation (1.1) has a kink profile solution

$$
\begin{aligned}
\Phi_{1}= & \int \frac{A\left(3 p^{3}+2-2 p\right)}{2(p+2) B\left(p^{3}+1-p\right)} \\
& \left.-\frac{2 h_{4}\left(3 p^{3}+2-2 p\right)}{p^{3} B(P+2)} \tanh ^{2}\left(\sqrt{h_{4}}\left(\xi+\xi_{0}\right)\right)\right]^{\frac{1}{p}} \mathrm{~d} \xi
\end{aligned}
$$

Th 3.2. 1) If

$$
\frac{p^{6} A^{2}}{16 h_{4}\left(p^{3}+1-p\right)^{2}}=\frac{h_{4}}{k^{2}},-\frac{A p^{3}}{2\left(p^{3}+1-p\right)}=-\frac{\left(1+k^{2}\right) h_{4}}{k^{2}}
$$

that Equation (1.1) has two Jacobian elliptic function solutions

$$
\begin{aligned}
\Phi_{2}= & \int \frac{A\left(3 p^{3}+2-2 p\right)}{2(p+2) B\left(p^{3}+1-p\right)} \\
& \left.-\frac{2 h_{4}\left(3 p^{3}+2-2 p\right)}{p^{3} B(P+2)} s n^{2}\left(\sqrt{\frac{h_{4}}{k^{2}}}\left(\xi+\xi_{0}\right)\right)\right]^{\frac{1}{p}} \mathrm{~d} \xi \\
\Phi_{3}= & \int \frac{A\left(3 p^{3}+2-2 p\right)}{2(p+2) B\left(p^{3}+1-p\right)} \\
& \left.-\frac{2 h_{4}\left(3 p^{3}+2-2 p\right)}{p^{3} B(P+2)} \frac{c n\left(\sqrt{\frac{h_{4}}{k^{2}}}\left(\xi+\xi_{0}\right)\right)}{d n\left(\sqrt{\frac{h_{4}}{k^{2}}}\left(\xi+\xi_{0}\right)\right)}\right]^{\frac{1}{p}} \mathrm{~d} \xi
\end{aligned}
$$

2) If

$$
\begin{aligned}
& \frac{p^{6} A^{2}}{16 h_{4}\left(p^{3}+1-p\right)^{2}}=-\frac{\left(k^{2}-1\right) h_{4}}{k^{2}}, \\
& -\frac{A p^{3}}{2\left(p^{3}+1-p\right)}=-\frac{\left(2-k^{2}\right) h_{4}}{k^{2}}
\end{aligned}
$$

that Equation (1.1) has a Jacobian elliptic function solution

$$
\begin{aligned}
\Phi_{4}= & \int \frac{A\left(3 p^{3}+2-2 p\right)}{2(p+2) B\left(p^{3}+1-p\right)} \\
& \left.-\frac{2 h_{4}\left(3 p^{3}+2-2 p\right)}{p^{3} B(P+2)} c n\left(\sqrt{-\frac{h_{4}}{k^{2}}}\left(\xi+\xi_{0}\right)\right)\right]^{\frac{1}{p}} \mathrm{~d} \xi
\end{aligned}
$$

3) If

$$
\begin{aligned}
& \frac{p^{6} A^{2}}{16 h_{4}\left(p^{3}+1-p\right)^{2}}=-\left(k^{2}-1\right) h_{4}, \\
& -\frac{A p^{3}}{2\left(p^{3}+1-p\right)}=-\left(2-k^{2}\right) h_{4}
\end{aligned}
$$

that Equation (1.1) has a Jacobian elliptic function solution

$$
\begin{aligned}
\Phi_{5}= & \int \frac{A\left(3 p^{3}+2-2 p\right)}{2(p+2) B\left(p^{3}+1-p\right)} \\
& \left.-\frac{2 h_{4}\left(3 p^{3}+2-2 p\right)}{p^{3} B(P+2)} d n\left(\sqrt{\frac{h_{4}}{k^{2}}}\left(\xi+\xi_{0}\right)\right)\right]^{\frac{1}{p}} \mathrm{~d} \xi
\end{aligned}
$$

4) If

$$
\frac{p^{6} A^{2}}{16 h_{4}\left(p^{3}+1-p\right)^{2}}=k^{2} h_{4},-\frac{A p^{3}}{2\left(p^{3}+1-p\right)}=-\left(k^{2}+1\right) h_{4}
$$

that Equation (1.1) has two Jacobian elliptic function solutions

$$
\begin{aligned}
\Phi_{6}= & \int \frac{A\left(3 p^{3}+2-2 p\right)}{2(p+2) B\left(p^{3}+1-p\right)} \\
& \left.-\frac{2 h_{4}\left(3 p^{3}+2-2 p\right)}{p^{3} B(P+2)} \frac{1}{s n\left(\sqrt{h_{4}}\left(\xi+\xi_{0}\right)\right)}\right]^{\frac{1}{p}} \mathrm{~d} \xi \\
\Phi_{7}= & \int\left[\frac{A\left(3 p^{3}+2-2 p\right)}{2(p+2) B\left(p^{3}+1-p\right)}\right. \\
& \left.-\frac{2 h_{4}\left(3 p^{3}+2-2 p\right)}{p^{3} B(P+2)} \frac{d n\left(\sqrt{h_{4}}\left(\xi+\xi_{0}\right)\right)}{c n\left(\sqrt{h_{4}}\left(\xi+\xi_{0}\right)\right)}\right]^{\frac{1}{p}} \mathrm{~d} \xi
\end{aligned}
$$

5) If

$\frac{p^{6} A^{2}}{16 h_{4}\left(p^{3}+1-p\right)^{2}}=-\frac{k^{2} h_{4}}{1-k^{2}},-\frac{A p^{3}}{2\left(p^{3}+1-p\right)}=\frac{\left(2 k^{2}-1\right) h_{4}}{1-k^{2}}$ that Equation (1.1) has a Jacobian elliptic function solution

$$
\begin{aligned}
\Phi_{8} & =\int \frac{A\left(3 p^{3}+2-2 p\right)}{2(p+2) B\left(p^{3}+1-p\right)} \\
& \left.-\frac{2 h_{4}\left(3 p^{3}+2-2 p\right)}{p^{3} B(P+2)} \frac{1}{c n\left(\sqrt{\frac{h_{4}}{1-k^{2}}}\left(\xi+\xi_{0}\right)\right.}\right]^{\frac{1}{p}} \mathrm{~d} \xi
\end{aligned}
$$


6) If

$\frac{p^{6} A^{2}}{16 h_{4}\left(p^{3}+1-p\right)^{2}}=-\frac{h_{4}}{k^{2}-1},-\frac{A p^{3}}{2\left(p^{3}+1-p\right)}=\frac{\left(2-k^{2}\right) h_{4}}{k^{2}-1}$

that Equation (1.1) has a Jacobian elliptic function solution

$$
\begin{aligned}
\Phi_{9}= & \int \frac{A\left(3 p^{3}+2-2 p\right)}{2(p+2) B\left(p^{3}+1-p\right)} \\
& \left.-\frac{2 h_{4}\left(3 p^{3}+2-2 p\right)}{p^{3} B(P+2)} \frac{1}{d n\left(\sqrt{\frac{h_{4}}{k^{2}-1}}\left(\xi+\xi_{0}\right)\right)}\right]^{\frac{1}{p}} \mathrm{~d} \xi
\end{aligned}
$$

7) If

$$
\begin{aligned}
& \frac{p^{6} A^{2}}{16 h_{4}\left(p^{3}+1-p\right)^{2}}=\left(1-k^{2}\right) h_{4}, \\
& -\frac{A p^{3}}{2\left(p^{3}+1-p\right)}=\left(2-k^{2}\right) h_{4}
\end{aligned}
$$

that Equation (1.1) has a Jacobian elliptic function solution

$$
\begin{aligned}
\Phi_{10}= & \int \frac{A\left(3 p^{3}+2-2 p\right)}{2(p+2) B\left(p^{3}+1-p\right)} \\
& \left.-\frac{2 h_{4}\left(3 p^{3}+2-2 p\right)}{p^{3} B(P+2)} \frac{c n\left(\sqrt{h_{4}}\left(\xi+\xi_{0}\right)\right)}{\operatorname{sn}\left(\sqrt{h_{4}}\left(\xi+\xi_{0}\right)\right)}\right]^{\frac{1}{p}} \mathrm{~d} \xi
\end{aligned}
$$

8) If

$\frac{p^{6} A^{2}}{16 h_{4}\left(p^{3}+1-p\right)^{2}}=\frac{h_{4}}{1-k^{2}},-\frac{A p^{3}}{2\left(p^{3}+1-p\right)}=\frac{\left(2-k^{2}\right) h_{4}}{1-k^{2}}$

that Equation (1.1) has a Jacobian elliptic function solution

$$
\begin{aligned}
\Phi_{11} & =\int \frac{A\left(3 p^{3}+2-2 p\right)}{2(p+2) B\left(p^{3}+1-p\right)} \\
& \left.-\frac{2 h_{4}\left(3 p^{3}+2-2 p\right)}{p^{3} B(P+2)} \frac{s n\left(\sqrt{\frac{h_{4}}{1-k^{2}}}\left(\xi+\xi_{0}\right)\right)}{c n\left(\sqrt{\frac{h_{4}}{1-k^{2}}}\left(\xi+\xi_{0}\right)\right.}\right]^{\frac{1}{p}} \mathrm{~d} \xi
\end{aligned}
$$

9) If

$$
\begin{aligned}
& \frac{p^{6} A^{2}}{16 h_{4}\left(p^{3}+1-p\right)^{2}}=\frac{h_{4}}{k^{2}\left(k^{2}-1\right)}, \\
& -\frac{A p^{3}}{2\left(p^{3}+1-p\right)}=\frac{\left(2 k^{2}-1\right) h_{4}}{k^{2}\left(k^{2}-1\right)}
\end{aligned}
$$

that Equation (1.1) has a Jacobian elliptic function solution

$$
\begin{aligned}
\Phi_{12} & =\int \frac{A\left(3 p^{3}+2-2 p\right)}{2(p+2) B\left(p^{3}+1-p\right)} \\
& \left.\left.-\frac{2 h_{4}\left(3 p^{3}+2-2 p\right)}{p^{3} B(P+2)} \frac{s n\left(\sqrt{\frac{h_{4}}{k^{2}\left(k^{2}-1\right)}}\left(\xi+\xi_{0}\right)\right)}{d n\left(\sqrt{\frac{h_{4}}{k^{2}\left(k^{2}-1\right)}}\left(\xi+\xi_{0}\right)\right.}\right)\right]^{\frac{1}{p}} \mathrm{~d} \xi
\end{aligned}
$$

10) If

$$
\begin{aligned}
& \frac{p^{6} A^{2}}{16 h_{4}\left(p^{3}+1-p\right)^{2}}=k^{2}\left(k^{2}-1\right) h_{4}, \\
& -\frac{A p^{3}}{2\left(p^{3}+1-p\right)}=\left(2 k^{2}-1\right) h_{4}
\end{aligned}
$$

that Equation (1.1) has a Jacobian elliptic function solution

$$
\begin{aligned}
\Phi_{13}= & \int\left[\frac{A\left(3 p^{3}+2-2 p\right)}{2(p+2) B\left(p^{3}+1-p\right)}\right. \\
& \left.-\frac{2 h_{4}\left(3 p^{3}+2-2 p\right)}{p^{3} B(P+2)} \frac{d n\left(\sqrt{h_{4}}\left(\xi+\xi_{0}\right)\right)}{\operatorname{sn}\left(\sqrt{h_{4}}\left(\xi+\xi_{0}\right)\right)}\right]^{\frac{1}{p}} \mathrm{~d} \xi
\end{aligned}
$$

11) If

$$
\frac{p^{6} A^{2}}{16 h_{4}\left(p^{3}+1-p\right)^{2}}=h_{4},-\frac{A p^{3}}{2\left(p^{3}+1-p\right)}=2\left(1-2 k^{2}\right) h_{4}
$$

that Equation (1.1) has four Jacobian elliptic function solutions

$$
\begin{aligned}
\Phi_{14}= & \int\left[\frac{A\left(3 p^{3}+2-2 p\right)}{2(p+2) B\left(p^{3}+1-p\right)}-\frac{2 h_{4}\left(3 p^{3}+2-2 p\right)}{p^{3} B(P+2)}\right. \\
& \left.\cdot\left[\frac{1}{s n \sqrt{4 h_{4}}\left(\xi+\xi_{0}\right)} \pm \frac{c n \sqrt{4 h_{4}}\left(\xi+\xi_{0}\right)}{s n \sqrt{4 h_{4}}\left(\xi+\xi_{0}\right)}\right]\right]^{\frac{1}{p}} \mathrm{~d} \xi
\end{aligned}
$$




$$
\begin{aligned}
\Phi_{15}= & \int \frac{A\left(3 p^{3}+2-2 p\right)}{2(p+2) B\left(p^{3}+1-p\right)} \\
& \left.-\frac{2 h_{4}\left(3 p^{3}+2-2 p\right)}{p^{3} B(P+2)}\left[\frac{s n \sqrt{4 h_{4}}\left(\xi+\xi_{0}\right)}{1 \pm c n \sqrt{4 h_{4}}\left(\xi+\xi_{0}\right)}\right]\right]^{\frac{1}{p}} \mathrm{~d} \xi \\
\Phi_{16}= & \int\left[\frac{A\left(3 p^{3}+2-2 p\right)}{2(p+2) B\left(p^{3}+1-p\right)}-\frac{2 h_{4}\left(3 p^{3}+2-2 p\right)}{p^{3} B(P+2)}\right. \\
& \left.\cdot\left[k s n \sqrt{4 h_{4}}\left(\xi+\xi_{0}\right) \pm i d n \sqrt{4 h_{4}}\left(\xi+\xi_{0}\right)\right]\right]^{\frac{1}{p}} \mathrm{~d} \xi \\
\Phi_{17}= & {\left[\frac{A\left(3 p^{3}+2-2 p\right)}{2(p+2) B\left(p^{3}+1-p\right)}-\frac{2 h_{4}\left(3 p^{3}+2-2 p\right)}{p^{3} B(P+2)}\right.} \\
& \left.\cdot\left[\frac{d n \sqrt{4 h_{4}}\left(\xi+\xi_{0}\right)}{k c n \sqrt{4 h_{4}}\left(\xi+\xi_{0}\right) \pm i \sqrt{1-m^{2}}}\right]\right]^{\frac{1}{p}} \mathrm{~d} \xi
\end{aligned}
$$

12) If

$$
\frac{p^{6} A^{2}}{16 h_{4}\left(p^{3}+1-p\right)^{2}}=h_{4},-\frac{A p^{3}}{2\left(p^{3}+1-p\right)}=\frac{4\left(1+k^{2}\right) h_{4}}{2\left(1-k^{2}\right)}
$$

that Equation (1.1) has a Jacobian elliptic function solution

$$
\begin{aligned}
\Phi_{18}= & \int \frac{A\left(3 p^{3}+2-2 p\right)}{2(p+2) B\left(p^{3}+1-p\right)} \\
& \left.-\frac{2 h_{4}\left(3 p^{3}+2-2 p\right)}{p^{3} B(P+2)}\left[\frac{c n \sqrt{\frac{4 h_{4}}{1-k^{2}}}\left(\xi+\xi_{0}\right)}{1 \pm s n \sqrt{\frac{4 h_{4}}{1-k^{2}}}\left(\xi+\xi_{0}\right)}\right]\right]^{\frac{1}{p}} \mathrm{~d} \xi
\end{aligned}
$$

13) If

$$
\begin{aligned}
& \frac{p^{6} A^{2}}{16 h_{4}\left(p^{3}+1-p\right)^{2}}=h_{4}, \\
& -\frac{A p^{3}}{2\left(p^{3}+1-p\right)}=\frac{2\left(k^{2}-2\right) h_{4}}{k^{2}}
\end{aligned}
$$

that Equation (1.1) has three Jacobian elliptic function solutions

$$
\begin{aligned}
\Phi_{19}= & \int\left[\frac{A\left(3 p^{3}+2-2 p\right)}{2(p+2) B\left(p^{3}+1-p\right)}-\frac{2 h_{4}\left(3 p^{3}+2-2 p\right)}{p^{3} B(P+2)}\right. \\
& \left.\cdot\left[s n \sqrt{\frac{4 h_{4}}{k^{2}}}\left(\xi+\xi_{0}\right) \pm i c n \sqrt{\frac{4 h_{4}}{k^{2}}}\left(\xi+\xi_{0}\right)\right]\right]^{\frac{1}{p}} \mathrm{~d} \xi
\end{aligned}
$$

$$
\begin{gathered}
\Phi_{20}=\int\left[\frac{A\left(3 p^{3}+2-2 p\right)}{2(p+2) B\left(p^{3}+1-p\right)}-\frac{2 h_{4}\left(3 p^{3}+2-2 p\right)}{p^{3} B(P+2)}\right. \\
\left.\cdot\left[\frac{d n \sqrt{\frac{4 h_{4}}{k^{2}}}\left(\xi+\xi_{0}\right)}{i \sqrt{1-k^{2}} s n \sqrt{\frac{4 h_{4}}{k^{2}}}\left(\xi+\xi_{0}\right) \pm c n \sqrt{\frac{4 h_{4}}{k^{2}}}\left(\xi+\xi_{0}\right)}\right]\right]^{\frac{1}{p}} \mathrm{~d} \xi \\
\Phi_{21}=\int\left[\frac{A\left(3 p^{3}+2-2 p\right)}{2(p+2) B\left(p^{3}+1-p\right)}\right. \\
\left.-\frac{2 h_{4}\left(3 p^{3}+2-2 p\right)}{p^{3} B(P+2)}\left[\frac{k s n \sqrt{\frac{4 h_{4}}{k^{2}}}\left(\xi+\xi_{0}\right)}{1 \pm d n \sqrt{\frac{4 h_{4}}{k^{2}}}\left(\xi+\xi_{0}\right)}\right]\right]^{\frac{1}{p}} \mathrm{~d} \xi
\end{gathered}
$$

14) If

$$
\frac{p^{6} A^{2}}{16 h_{4}\left(p^{3}+1-p\right)^{2}}=h_{4},-\frac{A p^{3}}{2\left(p^{3}+1-p\right)}=\frac{2\left(k^{2}+1\right) h_{4}}{k^{2}-1}
$$

that Equation (1.1) has a Jacobian elliptic function solution

$$
\begin{aligned}
\Phi_{22}= & \int \frac{A\left(3 p^{3}+2-2 p\right)}{2(p+2) B\left(p^{3}+1-p\right)} \\
& \left.-\frac{2 h_{4}\left(3 p^{3}+2-2 p\right)}{p^{3} B(P+2)} \frac{d n \sqrt{\frac{4 h_{4}}{k^{2}-1}}\left(\xi+\xi_{0}\right)}{1 \pm k s n \sqrt{\frac{4 h_{4}}{k^{2}-1}}\left(\xi+\xi_{0}\right)}\right]^{\frac{1}{p}} \mathrm{~d} \xi
\end{aligned}
$$

15) If

$$
\begin{aligned}
& \frac{p^{6} A^{2}}{16 h_{4}\left(p^{3}+1-p\right)^{2}}=\left(1-k^{2}\right)^{2} h_{4}, \\
& -\frac{A p^{3}}{2\left(p^{3}+1-p\right)}=-2\left(k^{2}+1\right) h_{4}
\end{aligned}
$$

that Equation (1.1) has a Jacobian elliptic function solution

$$
\begin{aligned}
\Phi_{23}= & \int\left[\frac{A\left(3 p^{3}+2-2 p\right)}{2(p+2) B\left(p^{3}+1-p\right)}-\frac{2 h_{4}\left(3 p^{3}+2-2 p\right)}{p^{3} B(P+2)}\right. \\
& \left.\cdot\left[k c n \sqrt{-4 h_{4}}\left(\xi+\xi_{0}\right) \pm d n \sqrt{-4 h_{4}}\left(\xi+\xi_{0}\right)\right]\right]^{\frac{1}{p}} \mathrm{~d} \xi
\end{aligned}
$$


16) If

$$
\begin{aligned}
& \frac{p^{6} A^{2}}{16 h_{4}\left(p^{3}+1-p\right)^{2}}=\frac{h_{4}}{\left(1-k^{2}\right)^{2}}, \\
& -\frac{A p^{3}}{2\left(p^{3}+1-p\right)}=\frac{2\left(k^{2}+1\right) h_{4}}{\left(1-k^{2}\right)^{2}}
\end{aligned}
$$

that Equation (1.1) has a Jacobian elliptic function solution

$$
\begin{aligned}
& \Phi_{24}=\int\left[\frac{A\left(3 p^{3}+2-2 p\right)}{2(P+2) B\left(p^{3}+1-p\right)}-\frac{2 h_{4}\left(3 p^{3}+2-2 p\right)}{p^{3} B(P+2)}\right. \\
& \left.\frac{s n \sqrt{\frac{4 h_{4}}{\left(1-k^{2}\right)^{2}}}\left(\xi+\xi_{0}\right)}{d n \sqrt{\frac{4 h_{4}}{\left(1-k^{2}\right)^{2}}}\left(\xi+\xi_{0}\right) \pm c n \sqrt{\frac{4 h_{4}}{\left(1-k^{2}\right)^{2}}\left(\xi+\xi_{0}\right)}}\right]^{\frac{1}{p}} \mathrm{~d} \xi
\end{aligned}
$$

17) If

$$
\frac{p^{6} A^{2}}{16 h_{4}\left(p^{3}+1-p\right)^{2}}=\frac{h_{4}}{k^{4}},-\frac{A p^{3}}{2\left(p^{3}+1-p\right)}=\frac{2\left(k^{2}-2\right) h_{4}}{k^{4}}
$$

that Equation (1.1) has a Jacobian elliptic function solution

$$
\begin{aligned}
\Phi_{25}= & \int \frac{A\left(3 p^{3}+2-2 p\right)}{2(p+2) B\left(p^{3}+1-p\right)}-\frac{2 h_{4}\left(3 p^{3}+2-2 p\right)}{p^{3} B(P+2)} \\
& \left.\cdot \frac{c n \sqrt{\frac{4 h_{4}}{k^{4}}}\left(\xi+\xi_{0}\right)}{\sqrt{1-k^{2}} \pm d n \sqrt{\frac{4 h_{4}}{k^{4}}}\left(\xi+\xi_{0}\right)}\right]^{\frac{1}{p}} \mathrm{~d} \xi
\end{aligned}
$$

\section{Conclusion}

In the paper, we apply the auxiliary equation method to study Equation (1.1), and get some new conclusions. Some new exact traveling wave solution of Equation (1.1) are obtain which include new singular solution, triangular periodic wave solutions and Jacobian elliptic function solutions. These solutions may be useful for describing certain nonlinear physical phenomena of Equation (1.1). The method which we have propose in this paper is standard, direct and computerized method ,which allow us to do complicated and tedious algebraic calculation.It is shown that the algorithm can be also applied to other nonlinear wave equations such as generalized BBM equation,

$$
u_{t}+a u^{p} u_{x}+b u^{2 p}-\delta u_{x x t}=0
$$

and generalized Klein-Gordon equation:

$$
u_{t t}-k\left(u_{x x}+u_{y y}\right)+b_{1} u+b_{2}|u|^{2 p} u+b_{3}|u|^{4 p} u=0
$$

\section{REFERENCES}

[1] M. J. Ablowitz and P. A. Clarkson, "Solution, Nonlinearn Evolution Equations and Inverse Scateing,” Cambridge University Press, Cambridge, 1991. doi:10.1017/CBO9780511623998

[2] M. Wadati, "Wave Propagation in Nonlinear Lattice. I," Journal of the Physical Society of Japan, Vol. 38, No. 3, 1975, pp. 673-680. doi:10.1143/JPSJ.38.673

[3] M. Wadati, H. Sanuki and K. Konno, "A Generalization of Inverse Scattering Method," Journal of the Physical Society of Japan, Vol. 46, No. 6, 1975, pp. 1965-1966. doi:10.1143/JPSJ.46.1965

[4] V. B. Matveev and M. A. Salle, "Darboux Transformation and Solitons,” Springer, Berlin, 1991.

[5] C. H. Gu, H. S. Hu and Z. X. Zhou, "Darboux Transformation in Solition Thory and Its Geometric Applications," Shanghai Scientific \& Techincal Publishers, Shanghai, 1999.

[6] C. Rogers and W. K. Schief, "Bäcklund and Darboux Transformation, Geometry and Modern Application in Soliton,” Cambridge University Press, Cambridge, 2002. doi:10.1017/CBO9780511606359

[7] R. Hirota, “The Direct Method in Soliton Theroy,” Cambridge University Press, Cambidge, 2004.

[8] P. J. Olver, "Applications of Lie Groups to Differential Equations,” Springer, New York, 1993. doi:10.1007/978-1-4612-4350-2

[9] G. W. Bluman and S. Kumei "Symmetries and Differential Equations,” Springer, Berlin, 1989.

[10] J. R. Quintero, "Solitons and Periodic Traveling Waves for the 2D-Generalized Benney-Luke Equation," Applicable Analysis, Vol. 86, No. 3, 2007, pp. 331-351. doi:10.1080/00036810601152390 